\title{
Il supporto alla «leadership» diffusa in relazione agli esiti delle rilevazioni esterne
}

\section{Giovanni Moretti}

Università degli Studi "Roma Tre», Dipartimento di Scienze della Formazione

doi: 10.7358/ecps-2014-009-more_giovanni.moretti@uniroma3.it

\section{THE WEIGHT OF QUANTITATIVE STUDIES IN THE EXERCISING OF LEADERSHIP IN THE SCHOOL FIELD}

\section{Abstract}

The article presents the importance of leadership in educational policies and shows the weight of the spreading of outcomes of both national and international comparative assessments on leadership geared to promoting improvement processes. The contribution examines certain critical aspects of the Italian context that seem to limit the exercising of leadership in the school field: the use of survey results that make policymakers less accountable; the delusionary and reassuring use made of survey results for schools, teachers and school heads; the application of many external evaluation methods in the face of an evident disengagement with regard to the improvement of didactic organization and the qualification of learning processes. The article also indicates the spheres in which survey results are strategic in order to improve teaching and to qualify learning processes. Finally, the importance that leadership can have in the school field for promoting a culture of data and evaluation is affirmed.

Keywords: Educational policy, Evaluation, Improvement, International comparative assessments, Leadership.

\section{LA RILEVANZA DELLA «LEADERSHIP» NELLE POLITICHE EDUCATIVE}

A partire dal settembre 2000, con l'entrata in vigore del Regolamento sull'autonomia scolastica, in Italia si è deciso di superare definitivamente l'adozione 
dei modelli di leadership formale e gerarchica che hanno tradizionalmente caratterizzato il sistema di istruzione e formazione (Benadusi \& Serpieri, 2000; Benadusi \& Consoli, 2002). Successivamente il processo di costruzione dell'autonomia scolastica e la nuova organizzazione delle istituzioni scolastiche sono stati esplicitamente e sistematicamente orientati a promuovere per quanto più possibile l'esercizio della leadership educativa diffusa, soprattutto ai vari livelli del mesosistema e del microsistema scolastico. Con l'espressione leadership educativa diffusa ci si riferisce all'esercizio di una leadership fortemente focalizzata sul coinvolgimento diretto di tutti gli attori scolastici - con varie modalità di partecipazione e differenti assunzioni di responsabilità - nei processi decisionali e nel corso delle azioni che da quei processi hanno avvio (Earley \& Bubb, 2004; Barzanò, 2008; Serpieri, 2008; Domenici \& Moretti, 2011). Questa prospettiva considera la leadership come l'esito di relazioni e di processi comunicativi piuttosto che la specifica competenza che caratterizza la singola persona, e considera l'agire collaborativo, situato in un determinato contesto educativo, come un elemento in grado di qualificare più di altri il processo di costruzione e diffusione della leadership.

Il passaggio graduale al modello innovativo della leadership educativa diffusa se da una parte sembra non incontrare resistenze, registrando anzi una buona accoglienza nelle pratiche discorsive formali e informali presenti nel mondo della scuola, dall'altra incontra difficoltà nel tradursi operativamente all'interno di quei contesti culturali e lavorativi in cui molti degli attori coinvolti sono tradizionalmente abituati a delegare le responsabilità.

A fronte della notevole rilevanza attribuita alla leadership, tuttavia, né le politiche educative, né la specifica normativa primaria e secondaria che hanno regolamentato l'autonomia scolastica e la dirigenza scolastica, sono state in grado di individuare le misure necessarie per accompagnare e implementare lo sviluppo effettivo della leadership diffusa (Cavalli \& Fischer, 2012; Moretti, 2012). Possiamo dunque affermare che in Italia la leadership in ambito educativo, contrariamente a quanto si dichiara pubblicamente, non è promossa e non è supportata con strumenti e risorse adeguate e probabilmente è fortemente temuta per il suo potenziale innovativo. Nelle politiche educative spesso prevalgono gli approcci orientati al controllo, di tipo top-down, che sembrano poco rispettosi dell'autonomia scolastica e dell'identità culturale e progettuale delle istituzioni educative. Le politiche educative così facendo, invece di responsabilizzare i dirigenti e gli altri operatori scolastici, ad esempio motivandoli a raggiungere i risultati prefissati e ad innalzare la qualità dell'offerta formativa, hanno spesso provocato effetti deresponsabilizzanti nei confronti degli obiettivi da perseguire. La scarsa disponibilità di informazioni rappresenta uno dei fattori che più favorisce il mancato supporto alla leadership diffusa e all'assunzione delle responsabilità. Il presente contributo 
focalizza l'attenzione su un tipo particolare di informazioni, quelle fornite dagli esiti delle rilevazioni comparative (nazionali e internazionali) e delle indagini da parte di soggetti istituzionali esterni alla scuola. La disponibilità di tali informazioni e il loro uso efficace potrebbero consentire ai decisori politici di individuare con maggiore chiarezza le criticità riscontrate a livello di macrosistema e conseguentemente di stabilire alcune priorità di azione ritenute strategiche per farvi fronte. Per favorire il pieno esercizio della leadership diffusa a livello di meso e di microsistema, inoltre, i decisori politici dovrebbero preoccuparsi di assicurare le precondizioni indispensabili di tale esercizio, assegnando alle scuole le risorse necessarie per organizzare e progettare le attività educative in modo tale da raggiungere i traguardi prefissati. Allo stesso modo le istituzioni scolastiche dovrebbero avvalersi delle informazioni disponibili e richiederne delle ulteriori, soprattutto in forma disaggregata e contestualizzata, per meglio applicare nel proprio contesto di riferimento le priorità generali individuate a livello di macrosistema.

La disponibilità di informazioni affidabili a tutti i livelli potrebbe indubbiamente rivestire un'importante funzione di supporto alla promozione della leadership diffusa. Molte delle sfide educative attuali potrebbero essere affrontare con maggiore efficacia se tutti i soggetti fossero messi nella condizione di disporre di informazioni indispensabili per assumere decisioni consapevoli e per comprendere le ragioni delle altrui decisioni. Per innalzare la qualità dell'offerta formativa e per qualificare i processi interni alla scuola occorre in particolare che gli insegnanti e i dirigenti scolastici dispongano di solide basi di dati in grado di facilitare il confronto tra gli esiti delle rilevazioni esterne (nazionali, internazionali) con quelle interne.

Si potrebbe obiettare che non sempre la disponibilità di informazioni, in particolare la pubblicazione di informazioni riguardanti i risultati scolastici, favorisca un più efficace esercizio della leadership diffusa. Alcuni studi sugli effetti e sulle implicazioni derivanti dalla pubblicazione dei risultati scolastici, infatti, non evidenziano effetti positivi, tali da compensare gli alti costi sostenuti e soprattutto le conseguenze negative che si potrebbero profilare nel lungo periodo. Indagini che hanno preso in esame la pubblicazione dei risultati scolastici in Gran Bretagna e in Francia rilevano un'ampia gamma di criticità, che sono state sintetizzate in tre categorie: (a) problemi di ordine tecnico-analitico; (b) problemi di fruibilità dei dati; (c) problemi etico-politico-sociali. Gli effetti della pubblicazione degli indicatori di risultato sui genitori sono ancora poco indagati, tuttavia emerge che influenzano una parte delle famiglie di ceto medio o medio-alto, mentre le altre famiglie dichiarano di non conoscere i dati, di non comprenderli o di non avere comunque alternative percorribili. Gli effetti della pubblicazione degli indicatori di risultato sugli istituti mostrano che le scuole diventano più sensibili alle logiche di 
mercato e quindi si dimostrano più attente alla loro reputazione. Conseguentemente le scuole tendono ad adottare strategie diverse quali: attività promozionali, analisi ambientali, cambiamenti fondamentali nei curricoli, cambiamenti strutturali nell'amministrazione dell'istituto, cambiamenti nella gestione delle risorse (Karsten \& Visscher, 2003; Visscher, Bert Dijkstra, Karsten, \& Veenstra, 2003).

Nonostante le evidenze sopra ricordate, di cui si deve tenere conto soprattutto per prevenire eventuali comportamenti autoreferenziali o disfunzionali delle istituzioni scolastiche, non è accoglibile l'obiezione alla tesi che sostiene l'importanza dell'uso delle rilevazioni esterne per il supporto alla leadership diffusa. La disponibilità di un flusso continuo di informazioni e il loro utilizzo nei processi decisionali in ambito scolastico sono risorse indispensabili per favorire il coinvolgimento attivo e la condivisione delle responsabilità di tutti nel rispetto dei diversi ruoli e funzioni svolte.

In ogni caso è auspicabile che insieme alla scuola ci sia l'impegno di tutti i soggetti esterni interessati - governi, media, amministrazione scolastica, ricercatori, ecc. - per incrementare la qualità delle pubblicazioni degli esiti delle rilevazioni, per sviluppare standard condivisi per la pubblicazione degli indicatori di prestazione riguardo ai criteri di precisione, fruibilità e cautela, per prevenire in particolare eventuali effetti perversi o indesiderati che possono accrescere le disuguaglianze esistenti.

\section{AlCUNi OSTACOLI ALL'ESERCIZIO EFFETTIVO DELLA «LEADERSHIP» IN CAMPO SCOLASTICO}

In Italia siamo ancora assai lontani dalla implementazione di politiche educative evidence based, che individuano obiettivi di medio e lungo termine e effettuano delle scelte strategiche o tattiche, prendendo a riferimento gli esiti più accreditati della ricerca educativa nazionale e internazionale (Calvani, 2011; Domenici, 2011). Nel nostro Paese non solo si continua a diminuire colpevolmente l'entità delle risorse necessarie per promuovere la ricerca educativa e didattica (Domenici, 2011, 2012, 2013a, 2013b), ma si mantiene un profilo molto basso nella diffusione degli esiti delle rilevazioni internazionali PISA (Programme for International Student Assessment), e si fatica ad avviare una riflessione critica su di essi (Bottani, 2003; Siniscalco, 2003; Domingo, 2006; Asquini, 2011); si presentano inoltre gli esiti delle rilevazioni INVALSI (Istituto Nazionale per la Valutazione del Sistema dell'Istruzione) con modalità e piste interpretative spesso funzionali alle politiche del momento, ma talvolta disfunzionali per orientare l'opinione pubblica. 
Alcuni esperti presentano gli esiti delle rilevazioni comparative su larga scala invitando i lettori a interpretare i dati con prudenza e suggerendo loro di tenere in considerazione le caratteristiche specifiche dei differenti sistemi scolastici presi in esame. La comparazione, infatti, nel prendere in considerazione contesti socioculturali ed economici assai diversi favorisce il confronto attraverso l'individuazione di alcuni indici e parametri comuni, ma non tiene conto di alcune opzioni e dimensioni, che a livello di sistema sono considerate di notevole rilevanza (accoglienza/segregazione, cooperazione/ competizione, accesso/selezione, giustizia/ingiustizia, equità/disuguaglianza, ecc.). Tuttavia nonostante le riflessioni critiche circa la possibilità o meno di comparare tra loro differenti sistemi educativi, in molti concordano nel considerare insostituibili le indagini su larga scala (Bottani, 2003), ritenendole indispensabili per disporre delle evidenze empiriche utili per acquisire consapevolezza di quegli aspetti del sistema educativo che richiedono scelte preventive o la messa a punto di interventi regolativi.

Per questo, in molti Paesi, la pubblicazione dei risultati delle valutazioni su larga scala provoca discussioni e polemiche, ma soprattutto stimola analisi critiche, dibattiti mirati, e sollecita la volontà di approfondire le questioni ritenute più rilevanti per assumere decisioni e intervenire nel medio e nel lungo periodo, sia a livello di sistema scolastico complessivo che di singole istituzioni scolastiche. Molti esperti e commentatori, purtroppo, da lungo tempo denunciano la sistematica disattenzione dell'Italia riguardo agli esiti delle rilevazioni, che sono puntualmente ignorati o sottovalutati dai media, così come dai decisori politici e conseguentemente anche dal personale scolastico e dalla opinione pubblica. Pur facendo parte dei Paesi a economia avanzata, dunque, dobbiamo prendere atto che l'Italia si dimostra poco lungimirante in campo educativo, e che non fa ancora parte a pieno titolo di quei Paesi moderni che praticano l'innovazione e la ricerca e che si avvalgono delle rilevazioni su larga scala per capire $i$ modi in cui rendere più efficienti ed efficaci quegli ambiti del sistema scolastico che difettano per quantità o per talune qualità. I media e i decisori politici, in Italia, nei rari casi in cui hanno affrontato le questioni educative, hanno adottato un fare strumentale, più orientato alla difesa di opinioni e posizioni assunte in precedenza, che all'avvio di un confronto informato con tutti i soggetti interessati, le scuole in particolare, finalizzato alla individuazione di alcune priorità di intervento.

L'uso degli esiti delle rilevazioni comparative internazionali e di quelle condotte da soggetti istituzionali esterni sull'esercizio effettivo della leadership educativa in ambito scolastico è a oggi assai scarso. Il governo locale delle istituzioni scolastiche e il più ampio processo di autonomia scolastica, dunque, si avvalgono poco o per nulla delle informazioni comunque disponibili e che se utilizzate potrebbero aiutare a meglio indirizzare il governo 
della scuola, a migliorare l'organizzazione della didattica e dei processi di insegnamento-apprendimento.

In particolare possiamo individuare tre aspetti che paiono limitare notevolmente il peso effettivo degli esiti delle rilevazioni comparative e delle ricerche sui processi di miglioramento o che ne ostacolano la possibilità di incidere positivamente, orientando l'esercizio della leadership in campo scolastico in Italia:

- un utilizzo degli esiti delle rilevazioni che deresponsabilizza i decisori politici;

- l'utilizzo illusorio e rassicurante degli esiti delle rilevazioni per le scuole, gli insegnanti e i dirigenti;

- l'applicazione di molte modalità di valutazione esterna a fronte di un palese disimpegno in merito al miglioramento della organizzazione didattica e alla qualificazione dei processi di apprendimento.

Qui di seguito esaminiamo ciascuno degli aspetti sopra evidenziati.

\subsection{Utilizzo degli esiti delle rilevazioni che deresponsabilizza i decisori politici}

I principali destinatari degli esiti delle rilevazioni nazionali e internazionali dovrebbero essere i decisori politici, a loro infatti compete la scelta di stabilire le priorità del sistema di istruzione e formazione, di indicare gli indirizzi generali e specifici a partire dai quali, a livello locale, le scuole autonome e gli insegnanti, coerentemente con essi, dovrebbero progettare l'offerta formativa.

I destinatari effettivi degli esiti delle ricerche, invece, diventano troppo spesso le scuole, gli insegnanti e i dirigenti scolastici, soggetti ai quali la stampa, i nuovi mezzi di comunicazione sociale e i politici stessi attribuiscono impropriamente una serie di responsabilità di cui non sono titolari. In questo modo, operando un ribaltamento dei ruoli, si valutano i prodotti senza tenere conto delle condizioni di contesto e dei mezzi e si promuove una cultura della valutazione che rischia di colpevolizzare e mortificare i più deboli e deresponsabilizzare i decisori politici (Lucisano, 2010, 2011, 2012).

Chi organizza le rilevazioni non diffonde in modo adeguato i risultati rispetto ai potenziali utenti. A fronte di risultati effettivamente fruibili da molteplici destinatari, gli esiti (presenti nei rapporti e nei database) non sono efficacemente proposti e dunque neppure utilizzati per orientare in modo strategico e continuo le macro e le micro decisioni che i diversi soggetti sono chiamati a compiere ai vari livelli di responsabilità. La deresponsabilizzazione dei decisori politici è facilmente osservabile in particolare nei casi in cui i dati sono diffusi: - con modalità discorsive che li fanno percepire dai destinatari come fatti stabili o consolidati e non come eventi modificabili; 
- senza che siano accompagnati da indicazioni sulle priorità strategiche da adottare per migliorare il sistema scolastico nel suo complesso;

- omettendo di contestualizzare gli esiti facendo riferimento alle caratteristiche dei contesti di riferimento presi in esame e alle risorse messe a disposizione delle istituzioni scolastiche;

- senza descrivere quali processi interni alle scuole e alle classi ci si è impegnati ad attivare o a migliorare prima della rilevazione dei dati;

- dimenticando di indicare in modo chiaro i miglioramenti che si intendono perseguire nel breve, medio e lungo periodo a partire dai dati stessi.

Gli esiti delle rilevazioni sono spesso diffusi e commentati dai decisori politici sulla base delle loro convenienze tattiche e di breve periodo; sono inoltre utilizzati strumentalmente per consolidare nell'opinione pubblica l'idea errata che le responsabilità connesse al mancato raggiungimento dei risultati prefissati o auspicati nel campo dell'istruzione e della formazione siano da attribuirsi prevalentemente alle scuole, agli insegnanti e ai dirigenti scolastici. Degli esiti complessivi delle rilevazioni in campo scolastico si parla poco e quando lo si fa si enfatizzano singoli temi o si stigmatizzano aspetti specifici, alimentando in questo modo il fenomeno del populismo mediatico che rappresenta il supporto infrastrutturale del clima d'opinione contemporaneo (Mazzoleni, 2003; Grossi, 2009). Tale fenomeno spinge le persone a reagire emotivamente e in modo inconsapevole trascurando di discriminare tra i livelli effettivi entro cui si distribuiscono le responsabilità nel sistema scuola. Esistono due dimensioni del populismo mediatico che si intrecciano: da un lato lo sfondo contestuale che, facendo leva su una concezione della realtà riconducibile al "costruzionismo", determina orientamenti e appartenenze sui diversi aspetti della leadership diffusa; dall'altro, l'influenza che lo sfondo contestuale esercita sui processi decisionali dei diversi soggetti della leadership diffusa. Per esempio può accadere che gli insegnanti siano influenzati negativamente dai ripetuti messaggi che, a partire dalla interpretazione parziale dei dati disponibili, tendono ad attribuire loro le maggiori responsabilità circa la scarsa qualità del sistema scolastico, squalificando in questo modo l'immagine pubblica della professione docente. La consapevolezza della scarsa considerazione sociale a sua volta può demotivare gli insegnanti e conseguentemente limitarne la disponibilità ad assumersi compiti e responsabilità specifiche che non siano strettamente attinenti alle tradizionali attività di insegnamento-apprendimento. $\mathrm{O}$ ancora, quando i media semplificano la rappresentazione del processo di autonomia scolastica enfatizzando il ruolo del cosiddetto «preside-manager» e il suo presunto potere di trasformare da solo l'istituzione scolastica, adottano di fatto un approccio populista che nel ridurre la complessità dei problemi non aiuta l'opinione pubblica a capire la distribuzione effettiva delle responsabilità tra i diversi attori della scuola 
di oggi. Una ulteriore dimostrazione è la presentazione degli esiti delle rilevazioni offerta dai media che non tenendo conto della scarsa informazione della opinione pubblica, dagli interessi assai frammentati, lascia ai genitori la libertà di rappresentarsi l'ambiente scolastico frequentato dai figli riferendosi alla propria esperienza e non piuttosto avvalendosi delle osservazioni dirette, delle evidenze e dei dati disponibili.

Il recente dibattito sul New Realism può aiutarci a riportare al centro dell'attenzione «la questione relativa alla distinzione tra sapere accreditabile come scientifico e sapere altro, ovvero ai tanti modi di conoscere, pure di grande rilievo e dignità» (Domenici, 2011, p. 16). L'equivoco fondamentale del costruzionismo è stato quello di pensare che la realtà in quanto costruzione sociale non abbia forma senza l'azione di una costruzione concettuale e che il dato sia un mito. Dovremmo piuttosto imparare a distinguere, caso per caso, e con pazienza, tra ciò che è naturale e cosa è culturale, tra ciò che è costruito e cosa non lo è. Si tratta di coltivare un sano realismo, che da una parte ci ricorda che un mondo là fuori c'è, indipendentemente da noi, e che dall'altra ci invita a conoscerlo meglio, e capirlo. Assumendo questa nuova prospettiva è decisivo che le nostre interazioni e decisioni di merito siano registrate e sottoposte alla regola della "documentabilità» consentendone così l'accesso alla dimensione della oggettività e della intersoggettività, favorendo al tempo stesso la promozione della cultura del dato e della valutazione, questione di cui si tratta nel paragrafo quattro del presente contributo.

Leggere, capire e interpretare correttamente i dati disponibili da parte degli insegnanti, dei dirigenti scolastici, delle famiglie e degli studenti, diventa sempre più una delle precondizioni indispensabili per affrontare efficacemente le nuove sfide educative e progettare percorsi didattici innovativi in grado di coniugare il miglioramento con l'efficacia degli interventi educativi. Ricordiamo, infatti, che il framework iniziale di PISA (OECD, 1999, 2010a, $2010 \mathrm{~b}$ ) individua come destinatari delle rilevazioni comparative non solo i decisori politici e gli studiosi di pedagogia, ma tutti i soggetti implicati nella scuola: insegnanti, dirigenti scolastici, studenti e famiglie. Anche le ricerche INVALSI sono tra l'altro finalizzate al superamento dell'handicap informativo degli utenti della scuola e intenzionate a rendere più consapevoli le scelte delle famiglie e più convinta la loro pressione al miglioramento nei riguardi del servizio scolastico (Sestito, 2012).

Molte sono le ragioni che giustificano l'esigenza di presentare i dati con chiarezza per consentire a tutti i cittadini di comprendere la reale portata degli esiti delle rilevazioni e delle comparazioni nazionali e internazionali. Per questo motivo occorre evitare che i media diffondano le informazioni con titolazioni spesso sensazionalistiche, dimenticando di offrire al lettore gli elementi necessari per capire cosa sia effettivamente accaduto; occorre anche 
evitare di confezionare notizie ambigue e contraddittorie che enfatizzano allo stesso modo l'individuazione di alcuni elementi di miglioramento e il riscontro di insufficienze e carenze croniche (Asquini, 2011).

Il confronto diacronico dei dati, reso oggi possibile dalla disponibilità delle serie storiche, consente a tutti gli interessati di rilevare e analizzare in modo affidabile le tendenze in atto e di capire in che modo assecondarle o fronteggiarle nei differenti contesti educativi formali e non formali. Scoprire ogni tre anni, ovvero ogni ciclo di rilevazione PISA, che gli studenti italiani si collocano nelle posizioni basse della classifica dei Paesi ad economie avanzate nel possesso delle competenze ritenute indispensabili per la vita e per l'esercizio della cittadinanza attiva è un fatto che dovrebbe richiamare l'attenzione di tutti, dei decisori politici in primo luogo, e impegnare ciascuno per la propria parte ad attivare rimedi efficaci per consentire al sistema nel suo complesso di cambiare rotta. Gli esiti dell'indagine ISFOL-PIAAC (Programme for the International Assesment of Adult Competencies) promossa dall'OCSE in ventiquattro Paesi conferma i bassi livelli raggiunti dalla popolazione adulta italiana tra i 16 e i 65 anni nelle competenze alfabetiche (literacy) e in quelle matematiche (numeracy). Si tratta di evidenze importanti, che avrebbero dovuto stimolare l'avvio di discussioni e che avrebbero dovuto anche sollecitare i decisori politici ad individuare nuove strategie di formazione adeguate ad affrontare le notevoli criticità riscontrate (Coros, 2013; ISFOL, 2013).

Gli esiti pur dirompenti delle rilevazioni non sono stati presi in considerazione per mobilitare nuove risorse e per implementare più efficaci politiche educative, ma hanno ulteriormente alimentato la deresponsabilizzazione dei decisori politici che si sono adoperati per rinegoziare e abbassare la soglia degli obiettivi stabiliti nell'ambito delle politiche europee. Nella determinazione ufficiale dei traguardi nazionali del Programma «Europa 2020» in materia di ricerca e istruzione, infatti, l'Italia ha purtroppo fissato a $1.5 \%$ invece che a 3\% la crescita in Pil del suo investimento in Ricerca \& Sviluppo; al $15-16 \%$ la diminuzione degli abbandoni scolastici invece della loro riduzione sotto il 10\%; al 26-27\% il numero dei laureati tra i giovani 30-34enni invece di proporsi il superamento della soglia del 40\% (Domenici, 2013a e 2013b). Il mancato utilizzo degli esiti delle rilevazioni e la deresponsabilizzazione dei decisori politici ritardano il processo di modernizzazione di cui l'Italia ha estremamente bisogno e che dovrebbe individuare nella istruzione e formazione il volano principale su cui far leva per mobilitare le competenze, le motivazioni e le passioni di chi lavora nelle scuole. Si preferisce invece ridurre gli obiettivi e si tranquillizzano le scuole, gli insegnanti e i dirigenti scolastici sul fatto che le sfide educative possano essere affrontate introducendo qualche aggiustamento e adattamento, piuttosto che impegnandosi 
in processi innovativi di lungo periodo, ad esempio la digitalizzazione della didattica e lo sviluppo professionale continuo del personale, per citarne due tra i più urgenti.

\subsection{Utilizzo degli esiti delle rilevazioni che rassicura le scuole, gli insegnanti e i dirigenti scolastici}

Una parte considerevole delle politiche educative ha diffuso il mito delle prove oggettive ed ha promosso l'adozione del cosiddetto "stile INVALSI» nella strutturazione delle verifiche sommative da parte delle scuole e persino dell'editoria scolastica. A fronte del grave disagio provato dagli studenti e dagli insegnanti riguardo la somministrazione di test da parte dell'INVALSI, l'editoria scolastica ha risposto in modo propositivo, interpretando a suo modo un bisogno reale e proponendo quattro tipi di strumenti: libri di testo curricolari «adattati», libri specifici non adozionali, guide per l'insegnante arricchite di apparati ad hoc e strumenti multimediali on line (Favalli, 2011).

L'editoria, dunque, ha cercato di svolgere come sempre un ruolo di supplenza dovuto alla scarsa diffusione di una cultura della valutazione in ambito scolastico e tra i docenti, tuttavia essa ha favorito l'uso abnorme di testi di supporto, di guide, fascicoli e libretti, non sempre di qualità, progettati esclusivamente per allenare gli studenti ai test INVALSI. Materiali dai titoli più diversi, ad esempio Palestra INVALSI, Vacanze in rete. Prove INVALSI, sono stati adottati dagli insegnanti nel corso dell'anno scolastico e talvolta sono stati consigliati ai ragazzi, a integrazione o sostituzione dei tradizionali compiti per le vacanze (ibid.). La forma digitale di alcune proposte, facilmente accessibili online, si caratterizza per la presenza di dispositivi di autocorrezione, di risposte personalizzate e talvolta di sistemi di tutoraggio on line. L'autocorrezione in verità si limita ancora alla segnalazione delle risposte errate o corrette e solo in alcune proposte esplicita agli studenti i motivi che sono alla base delle risposte errate. Ritroviamo qui il prevalere delle funzioni valutative di tipo sommativo a discapito di quelle formative, che dovrebbero invece prestare attenzione alla qualità del feedback dato agli studenti per aiutarli ad acquisire la consapevolezza delle proprie strategie di ragionamento e dei propri processi di comprensione.

La questione più importante su cui riflettere non è tanto quella relativa all'alto o basso valore didattico dei materiali prodotti dalla editoria scolastica, ma al tipo di utilizzo che di tali materiali è fatto in ambito scolastico. E da questo punto di vista le criticità sono molteplici se a partire dalle prove si programmano le attività didattiche, si predispongono i compiti in classe, si calendarizzano le prove di verifica e si svolgono le esercitazioni in classe. 
Mentre in altri Paesi (ad esempio l'Olanda) la presenza di un nucleo centrale di esami e test parrebbe stimolare fra l'altro un «sano" teaching to the test, in Italia il mezzo rischia di trasformarsi progressivamente in un fine, quale è diventato l'addestramento intensivo al test, a discapito di quelle attività che dovrebbero invece aiutare gli studenti ad acquisire competenze riflessive e di problem solving richieste in particolare dalle prove PISA (Programme for International Student Assessment). La valutazione degli apprendimenti su larga scala attraverso prove strutturate è un'arma a doppio taglio per lottare contro le disuguaglianze perché l'uso di tali strumenti presenta sia vantaggi sia svantaggi di cui è opportuno tenere conto. Tra i vantaggi si riscontra la possibilità di: (a) fare confronti nel tempo e nello spazio e quindi di apprezzare gli effetti delle riforme scolastiche o dei programmi; (b) segnalare al pubblico gli obiettivi dell'educazione e dell'istruzione scolastiche che meritano di essere presi in considerazione; tra gli svantaggi, in particolare, si segnala che tali rilevazioni: (a) prendono in esame una gamma ristretta di competenze; (b) raccolgono informazioni di scarsa utilità per il lavoro in classe (Bottani, 2003, p. 52).

A fronte delle molte sfide educative che le scuole si trovano a fronteggiare, in particolare quelle riferite alla valorizzazione delle diversità intrapersonali e inter-personali degli alunni, il fenomeno della rassicurazione delle scuole, degli insegnanti e dei dirigenti scolastici è spesso associato all'uso riduttivo degli esiti delle rilevazioni che sposta l'attenzione sugli strumenti, a scapito della riflessione sulla revisione o riprogettazione delle attività didattiche. Altro fattore rassicurante è l'appiattimento dell'azione degli insegnanti e della scuola sull'uso esclusivo degli esiti delle prove ritenuti i principali riferimenti per la valutazione degli alunni e per governare efficacemente i processi di insegnamento-apprendimento. Un ulteriore uso rassicurante dei dati è quello di chi si avvale degli esiti delle rilevazioni più recenti ritenendo pregiudizialmente impossibile confrontarli con le serie storiche di dati raccolti in precedenza. Ladozione di approcci rassicuranti come quelli sopra esemplificati può essere gratificante perché nel ridurre la complessità delle questioni trattate favorisce l'illusione di tenere facilmente sotto controllo i processi scolastici avviati.

Le varie forme di utilizzo degli esiti delle rilevazioni e della ricerca con finalità rassicuranti per le scuole, gli insegnanti e i dirigenti possono mortificare la creatività e incoraggiare il conformismo, modello di comportamento, questo, in parte alimentato dai dispositivi adottati nella valutazione dei prodotti della ricerca. Tali dispositivi, infatti, hanno il potere di spingere, in modo irresistibile, non pochi ricercatori ad adottare modelli standard, a seguire procedure già note e meno rischiose, a puntare sulle idee tradizionali già esplorate in letteratura, a scegliere progetti sicuri a discapito di quelli in- 
novativi ma rischiosi (Albarea, 2013; Sylos Labini, 2013). Il nostro sistema scuola ha necessità di autonomia, di innovazione e di ricerca, ambiti questi che richiedono investimenti sistematici in una prospettiva temporale di medio e lungo periodo, perché il cambiamento effettivo in questi settori implica la messa in campo di processi che devono concorrere ad innalzare e diffondere la cultura progettuale, valutativa e didattica. Accade tuttavia che molte delle politiche di breve respiro messe in campo e le retoriche dei discorsi che le accompagnano sembrano scegliere scorciatoie e forme di decisionismo che puntano essenzialmente sulla figura del preside-manager e sulla individuazione di soluzioni tecniche di tipo gestionale-manageriale.

\subsection{Eccesso di valutazione esterna e disimpegno nelle azioni di miglioramento}

La valutazione scolastica è una risorsa soprattutto quando consente di assumere decisioni informate, permette di regolare l'azione didattica, favorisce l'individualizzazione dei processi di apprendimento e dedica attenzione alla verifica dell'impatto sugli alunni del processo di sviluppo professionale dello staff di supporto e dei docenti (Leithwood \& Day, 2008; Sammons, Gu, Day, \& Ko, 2011). La valutazione in campo educativo, viceversa, non costituisce una risorsa quando è principalmente finalizzata a classificare le scuole e distinguere gli alunni tra meritevoli e non meritevoli.

Lucisano afferma che "la maggior parte delle valutazioni a cui siamo esposti da parte di chi ha il potere di decidere e di fare opinione tende a basarsi su una analisi dei risultati che non tiene conto di uno o più passaggi e delle cautele scientifiche necessari. Il più comune dei fenomeni è quello di valutare i prodotti senza tenere conto delle condizioni di contesto e dei mezzi» (Lucisano, 2012, p. 15). Non meraviglia quindi che la valutazione esterna promossa dall'INVALSI appaia a molti satura di intenti moralistici e che rischi di colpevolizzare le scuole, gli insegnanti e i dirigenti scolastici, senza offrire loro indicazioni chiare in merito alle possibili azioni di miglioramento da intraprendere. Quello che emerge dalle indagini sin qui svolte è la contrarietà degli insegnanti per le forme di rendicontazione fortemente squilibrate sul versante della valutazione sommativa, intesa come controllo esterno e dunque vissuta come intrusiva e minacciosa per l'esercizio della propria professionalità. Tuttavia una parte degli insegnanti si dichiara disponibile ad impegnarsi in forme di autovalutazione prevalentemente orientate a valorizzare la valutazione formativa con finalità di miglioramento della organizzazione scolastica e dei processi di apprendimento.

D'altra parte l'autovalutazione d'istituto, quando è basata unicamente sui test, pone problemi di riconoscimento dell'impegno professionale dei do- 
centi, soprattutto di quelli impegnati in contesti difficili o svantaggiati oltre che problemi tecnici dovuti alla qualità delle prove, bersaglio di numerose critiche rivolte da membri autorevoli della comunità scientifica. L'introduzione della Prova Nazionale (PN) INVALSI nell'Esame di Stato al termine del I ciclo d'istruzione è un esempio emblematico di valutazione esterna che nelle intenzioni doveva rilevare i livelli di apprendimento degli studenti e favorire il progressivo allineamento a standard di carattere nazionale, ma che nella realtà si è rivelata per molti aspetti disfunzionale al raggiungimento di tali scopi, così come è dimostrato da alcune ricerche riguardanti il punto di vista specifico degli insegnanti di lettere. L'utilizzo degli esiti della PN sono esigui e strettamente funzionali all'assolvimento burocratico della prova stessa e trascura di migliorare ad esempio l'importante ambito della didattica della comprensione del testo (Marcuccio, 2010).

Tenuto conto che «In Italia, il programma di valutazione INVALSI può essere considerato un esempio di valutazione esterna orientata alla rendicontazione, con la prospettiva aggiuntiva che le scuole usino i risultati per scopi di autovalutazione» (Scheerens, 2011, p. 65) è opportuno promuovere indagini sull'uso dei dati restituiti dall'INVALSI e sulla loro integrazione con i sistemi di autovalutazione di istituto (Domenici \& Lucisano, 2011). L'integrazione tra fonti di dati, infatti, è particolarmente efficace quando si avvale della «analisi comparata dei dati valutativi prodotti da singole classi, scuole (autovalutazione d'istituto) e reti di scuole, e dei dati valutativi che potrà fornire l'INVALSI attraverso rapporti ad hoc, flussi e stock informativi quanto meno riferiti agli anni conclusivi dei cicli scolastici e a livello di aggregazione nazionale e regionale. Con rilevazioni INVALSI su base campionaria, ogni scuola, ogni classe potrebbe traguardare taluni aspetti del proprio operato con gli esiti più generali, impiegando autonomamente gli stessi strumenti delle rilevazioni nazionali» (Domenici, 2012, p. 82).

Forse una maggiore considerazione da parte dei decisori politici del possibile contributo che potrebbe dare la comunità scientifica dei pedagogisti consentirebbe di valorizzare e potenziare quella tradizione italiana che ha prodotto una vasta e articolata gamma di strumenti di autovalutazione e che è stata particolarmente sensibile nel rilevare il punto di vista espresso dai diversi attori scolastici. Tale tradizione, infatti, ha perseguito sistematicamente l'integrazione tra dimensione macro della valutazione e dimensione processuale e micro, prevenendo i rischi, sempre presenti, della loro separazione (Cardarello, 2012; Galliani, 2013).

L'Italia, ad oggi, rientra in quei Paesi «in cui gli accordi di rendicontazione consistono, soprattutto, in politiche di intenzioni non ancora pienamente realizzate; ci si aspetta tuttavia che i risultati della valutazione indirizzino l'autovalutazione della scuola» (Scheerens, 2011, p. 77). In questa prospetti- 
va che riconosce la rilevanza strategica dell'autovalutazione della scuola, l'individuazione di un alto profilo di leadership può essere definita in particolare da due indicatori: il grado di autorità decisionale e il grado in cui la leadership è di tipo educativo o trasformazionale piuttosto che tradizionale. Uno studio esplorativo condotto nella scuola primaria mette in evidenza il ruolo positivo svolto dalla valutazione a sostegno dell'apprendimento, ritenuta un dispositivo che responsabilizza tutti gli attori, in particolare i docenti e gli studenti, nella regolazione dei processi di apprendimento e nella co-costruzione attiva delle conoscenze (Giovannini \& Boni, 2010). La valutazione per l'apprendimento richiede la responsabilizzazione di tutti e implica anche l'esercizio della leadership diffusa degli studenti, che sono costantemente sollecitati a prestare attenzione agli aspetti metacognitivi e più in generale alla qualità dei propri processi di comprensione.

\section{AMBITI STRATEGICI PER IL MIGLIORAMENTO DELLA DIDATTICA E DEI PROCESSI DI APPRENDIMENTO}

In Svizzera, in Olanda e Germania, volendo citare alcuni dei Paesi a economia avanzata, le prove PISA e altre rilevazioni internazionali hanno stimolato studi "sui processi di insegnamento connessi», che focalizzano l'attenzione sul nesso tra risultati, processi e azioni didattiche svolte, e avviano diverse riflessioni sulle specifiche azioni didattiche che è possibile realizzare in modo efficace in un determinato contesto educativo (Cardarello, 2012). Mediante questo approccio la ricerca educativa può approfondire le connessioni tra dimensioni macro e micro, e può rilevare anche l'impatto che hanno alcune variabili strutturali dell'organizzazione scolastica su alcune variabili di processo (per esempio l'impatto della numerosità delle classi sulle relazioni docentistudenti intesa come coinvolgimento e partecipazione degli studenti). La ricerca educativa e didattica, anche di tipo locale, può aiutare a collegare gli esiti di apprendimento ad alcune strategie o pratiche didattiche, le cui categorizzazioni spesso si limitano a indicare il cosa (per esempio lettura a voce alta dell'insegnante), ma non sono in grado di specificare il come si configura nella "pratica». Nella presentazione delle ricerche comparative e più in generale nella letteratura internazionale è spesso presente il grave problema della cosiddetta «opacità degli interventi didattici» (ibid.), dovuta alla scarsa trasparenza in merito ai tratti salienti, cruciali, irrinunciabili o specifici che dicono a quali condizioni e con quali strumenti le strategie utilizzate possono avere un impatto positivo. Al tempo stesso è anche opportuno fare in modo che le azioni di miglioramento facilitino il confronto e l'accumulo delle co- 
noscenze, evitando di cedere alla diffusa tendenza che cerca di trasformare le strategie didattiche in "pacchetti pronti per l'uso».

L'uso degli esiti delle rilevazioni comparative nazionali e internazionali nell'esercizio della leadership in campo educativo dovrebbe aiutare ad individuare gli ambiti strategici su cui puntare per migliorare l'organizzazione della didattica e innalzare la qualità dei processi di apprendimento. Un aspetto rilevante su cui intervenire con urgenza è la promozione della leadership degli insegnanti, e in particolare l'organizzazione efficace dei Consigli di classe, dei Dipartimenti disciplinari o interdisciplinari e di team funzionali coesi e collaborativi, ma più in generale, la messa a punto di un sistema integrato di formazione continua e di sviluppo professionale dei docenti.

Tale sistema dovrebbe assumere come punto di riferimento principale il contesto lavorativo degli insegnanti, che è l'ambiente in cui essi svolgono le pratiche didattiche ordinarie. Un sistema che dovrebbe cercare di promuovere atteggiamenti riflessivi, anche decidendo di sottoporre alcuni processi interni alla scuola o alla classe al vaglio critico dei pari, del dirigente scolastico o di esperti esterni capaci di restituire agli interessati indicazioni orientate al miglioramento delle pratiche professionali. È a questo livello, che è il campo specifico in cui possiamo considerare l'insegnamento come attività altamente specializzata niente affatto intuitiva o da affidare all'improvvisazione, che dovremmo favorire l'aggancio tra piano concettuale, esiti delle rilevazioni e pratica didattica ordinaria (Pavan De Gregorio, 2003), favorendo l'innovazione e la spinta alla modifica delle proprie abitudini lavorative.

L'ipotesi di una leadership sostenibile, responsabile e diffusa (Hargreaves, 2007; Albarea, 2013), esercitata da dirigenti scolastici e dagli insegnanti, capace di individuare un criterio soglia, un punto di equilibrio da prendere a riferimento per coniugare la tradizione con la spinta al cambiamento, la riproposizione di pratiche didattiche consolidate con il "disapprendimento» di alcune di esse, è una risorsa strategica per favorire nel contesto scolastico il cambiamento effettivo e profondo delle convinzioni personali, delle credenze e dei modi in cui gli insegnanti organizzano la didattica.

È opportuno ricordare infatti che l'impatto più evidente delle prime indagini quantitative internazionali è stato sul modo di pensare il curricolo. $\mathrm{Si}$ è avuta la conferma e in taluni casi si è scoperta la forbice esistente tra "curricolo formale» e "curricolo insegnato», tra curricolo valutato e curricolo appreso dagli studenti. Riflettere su tali questioni potrebbe essere particolarmente utile per l'Italia, Paese in cui sappiamo ancora poco di quello che effettivamente si fa nelle scuole, dei modi in cui operano gli insegnanti, di ciò che pensano della loro professione e dei processi di apprendimento degli alunni.

Da più parti si segnala il rischio che la pratica lavorativa diffusa degli insegnanti resti ancorata ad una visione che pone al centro la lezione tradi- 
zionale e l'uso dei libri di testo scolastici, nonostante l'elaborazione del Piano dell'offerta formativa e malgrado l'introduzione dei nuovi curricoli basati sulle competenze (Cavalli, \& Argentin, 2010; Fondazione Giovanni Agnelli, 2011 e 2012). La maggior parte delle evidenze emerse dalle indagini comparative nazionali e internazionali è considerata estranea all'agire quotidiano dei contesti formativi o è ritenuta interessante ma inapplicabile nel proprio contesto scolastico. Questo atteggiamento di chiusura nei confronti delle novità indubbiamente non può essere addebitato alle scuole $\mathrm{o}$ ai singoli docenti e dirigenti. Abbiamo a che fare con una situazione dovuta alla cronica disattenzione ai temi della istruzione e della formazione, intendendo con questo, non tanto la pratica continua degli annunci innovativi o l'inizio di grandi riforme calate dall'alto, bensì l'avvio di un confronto continuo sulle questioni educative con argomentazioni basate su dati affidabili, guidate da esiti di monitoraggi e diagnosi condotti in modo rigoroso e trasparente.

La rendicontazione pubblica è connessa alle azioni di miglioramento della scuola e può essere effettuata avvalendosi di più fonti informative: (a) dati derivanti da test e prove di accertamento; (b) dati amministrativi e statistiche; (c) analisi e indagini sistematiche, ad esempio le ispezioni e l'autovalutazione d'istituto (Scheerens, 2011, p. 53). La prospettiva migliorativa contiene sempre elementi di valutazione formativa e proattiva; viceversa la prospettiva valutativa orientata alla rendicontazione fa prevalere gli elementi sommativi della valutazione (classifiche, controlli, premi, sanzioni, ecc.), motivo per cui è spesso considerata come minacciosa o ingiusta. La valutazione scolastica è dunque sottoposta a distinte pressioni, quella esterna finalizzata alla rendicontazione e quella interna per promuovere il miglioramento. Assumendo una prospettiva che integri le due pressioni potremo affermare che l'autovalutazione della scuola può essere considerata una forma decentrata di rendicontazione pubblica.

Alcune strategie più comuni di miglioramento sono inique (ad esempio: negare le iscrizioni agli studenti con problemi e con difficoltà di apprendimento; non ammettere gli studenti agli esami nazionali, ecc.), perché sono funzionali al miglioramento della posizione della scuola nelle classifiche e non invece a migliorare la qualità della didattica.

Di fronte alla domanda "Che cosa migliorare? Da quale aspetti iniziare?» è irrealistico pensare che le scuole individuino la risposta a partire da una sola idea di giustizia. Nelle scuole convivono più idee di giustizia, per cui è bene domandarci se e come il sistema nel suo insieme è integrato e coerente, e soprattutto se viola o meno i principi di equità e di giustizia nella vita quotidiana. Alcuni comportamenti delle scuole talvolta contraddicono il principio dell'equità di trattamento e alcune minacce all'equità dell'istruzione derivano persino dal processo di autonomia scolastica, perché rischia 
di esporre le scuole alla violazione di principi di giustizia educativa attraverso: (a) la segregazione scolastica; (b) la speculazione sugli alunni disabili; (c) la costituzione di reti di scuole per ripartirsi tra loro gli studenti (Bottani, 2003).

La garanzia dell'equità, per la scuola dell'autonomia, è una sfida, che esige risposte efficaci, basate su dati e informazioni affidabili, elementi oggi indispensabili per conoscere approfonditamente la direzione dei processi educativi. Affinché la qualificazione della didattica e l'innalzamento della qualità dei processi di apprendimento possano coniugarsi con l'equità e con il miglioramento complessivo della qualità del sistema di istruzione, è dunque auspicabile che la leadership educativa diffusa sia esercitata anche attraverso la promozione della cultura valutativa e l'uso responsabile del dato.

\section{4. "LEADERSHIP» IN CAMPO SCOLASTICO}

E PROMOZIONE DELLA CULTURA DEL DATO E DELLA VALUTAZIONE

Negli ultimi anni sono notevolmente aumentati sia i dati generati dal sistema scolastico (registri elettronici, anagrafe alunni, finanziamenti, reclutamento e mobilità del personale scolastico, ecc.) sia quelli raccolti dalle varie agenzie sui diversi livelli del sistema educativo. Questa ampia mole di dati, attualmente frammentata e solo in parte accessibile, grazie ai processi di digitalizzazione in corso rappresenta un'importante risorsa per l'intero sistema di istruzione e formazione. Le informazioni raccolte, provenienti da fonti differenti, infatti, già oggi sono in buona parte integrabili tra loro, consentendo a chi fosse interessato ad utilizzarle di generare nuovi dati e di fare elaborazioni specifiche e interpretazioni originali.

Il sistema educativo in Italia si trova impreparato ad affrontare la sfida della promozione della cultura del dato e della valutazione tra tutti gli attori scolastici, e tuttavia questa sfida va accolta perché è indispensabile fare in modo che il mondo della scuola si orienti in modo consapevole dentro il nuovo ambiente informativo globalizzato. Per raggiungere questo obiettivo può essere molto importante il ruolo svolto dalla leadership scolastica, da ritenersi strategico per aiutare gli insegnanti e i genitori ad avvalersi dei dati informativi oggi disponibili e superare possibili atteggiamenti di ostilità e di rifiuto. Gli esiti di una ricerca empirica esplorativa condotta in provincia di Bologna sulla restituzione e sull'utilizzo dei dati delle rilevazioni effettuate sia da soggetti esterni agli istituti scolastici (INVALSI, Osservatorio scolastico provinciale e Provincia), sia in forma autonoma dall'istituto scolastico, mostrano che all'interno di una cultura del dato e della valutazione sta emer- 
gendo, sia pure in modo diversificato e trasversale sul territorio e tra i diversi indirizzi scolastici, l'impatto abbastanza positivo delle rilevazioni realizzate dai soggetti esterni. Permane tuttavia la presenza di un certo numero di istituti in cui sembra rilevarsi un atteggiamento ambivalente e/o indifferente verso la cultura del dato e della valutazione, espressione di un certo disorientamento o di una chiusura autoreferenziale (Giovannini \& Marcuccio, 2007). In sintesi gli aspetti rilevanti emersi dall'analisi dei punti di vista dei diversi soggetti ascoltati che possono costituire un punto di partenza per un ulteriore sviluppo della cultura del dato e della valutazione sono:

1. concezione della scuola e dell'istituto scolastico come sottosistema sociale in interazione con un sistema sociale più complesso verso cui si è responsabili; 2. concezione della restituzione dei dati come fenomeno complesso in cui interagiscono molteplici variabili; 3 . influenza delle procedure e strumenti di progettazione e realizzazione delle rilevazioni sulla validità e attendibilità dei dati; 4. funzione formativa della restituzioni; 5 . modalità con cui gli insegnanti prendono le decisioni riguardo all'insegnamento e all'apprendimento; 6 . forme e modelli di rilevazione partecipate. (Giovannini, 2007, p. 156)

È dunque opportuno favorire l'utilizzo dei dati disponibili da parte di tutti i soggetti, istituzionali e non, interessati all'innalzamento della qualità dell'istruzione. I vincoli normativi attuali e la scarsa cultura della valutazione hanno impedito l'accesso ai molti dati oggi disponibili, la cui elaborazione, ad esempio, potrebbe aprire nuove strade per sviluppare modelli di valore aggiunto delle scuole, o costruire indicatori della situazione economica, sociale e culturale dello studente, o più in generale delle caratteristiche individuali degli studenti. L'accesso facilitato ai dati, inoltre, aiuterebbe a superare le limitazioni metodologiche e statistiche da più parti segnalate riguardo ad alcune scelte effettuate dall'INVALSI o dalla Fondazione Agnelli nella definizione di parametri, di indicatori e delle modalità seguite per confrontare $\mathrm{i}$ dati raccolti (Agasisti \& Sibiano, 2012).

La promozione della cultura del dato e della valutazione insieme alla dimensione didattica, considerata predominante, dovrebbe privilegiare anche quella strategica-organizzativa, troppo spesso dimenticata, che richiede la capacità di connettere, interrogare e far dialogare tra loro dati e informazioni (Giovannini, 2007; Domenici \& Lucisano, 2011b). 


\section{RIFERIMENTI BIBLIOGRAFICI}

Agasisti, T., \& Sibiano, P. (2012). Valutare le scuole italiane: osservazioni critiche sulle classifiche di Fondazione Agnelli. Statistica \& Società, 1(2), 25-26.

Albarea, R. (2013). Saperi esperienziali e leadership sostenibile nell'educazione degli adulti. Riflessioni pedagogiche. Journal of Educational, Cultural and Psychological Studies, 7, 161-186.

Asquini, G. (2011). Dieci anni di PISA: primi bilanci e nuove prospettive. Giornale Italiano della Ricerca Educativa, 4(7), 71-83.

Barzanò, G. (2008). Leadership per l'educazione. Riflessioni e prospettive dal dibattito globale. Roma: Armando.

Benadusi, L., \& Consoli, F. (a cura di). (2002). La governance della scuola. Istituzioni e soggetti alla prova dell'autonomia. Bologna: Il Mulino.

Benadusi, L., \& Serpieri, R. (a cura di). (2000). Organizzare la scuola dell'autonomia. Roma: Carocci.

Bottani, N. (2003). Le indagini comparate internazionali sul profitto scolastico. In N. Bottani \& A. Cenerini (a cura di), Una pagella per la scuola. La valutazione tra autonomia e qualità (pp. 265-287). Trento: Erickson.

Calvani, A. (2011). «Decision making» nell' istruzione. «Evidence based education»e conoscenze sfidanti. Journal of Educational, Cultural and Psychological Studies, 3, 77-99.

Cardarello, R. (2012). Ricerca didattica e valutazione. Giornale Italiano della Ricerca Educativa, Numero speciale, 5, 21-26.

Cavalli, A., \& Argentin, G. (a cura di). (2010). Gli insegnanti italiani: come cambia il modo di fare scuola. Terza indagine dell'Istituto IARD sulle condizioni di vita e di lavoro nella scuola italiana. Bologna: Il Mulino.

Cavalli, A., \& Fischer, L. (a cura di). (2012). Dirigere le scuole oggi. Bologna: Il Mulino.

Coros, C. (2013). Indagine PIAAC. L'Italia non sa leggere né far di conto. Nuova Secondaria, 31(6), 26-28.

Domenici, G. (2011). Strategia «Europa 2020», ricerca educativa e qualità della formazione. Journal of Educational, Cultural and Psychological Studies, 4, 9-16.

Domenici, G. (2012). Valutazione e autovalutazione come risorse aggiuntive nei processi di istruzione. Education Sciences \& Society, 2(2), 69-82.

Domenici, G. (2013a). Il declino (inarrestabile!?) dell'università e della ricerca in Italia. Journal of Educational, Cultural and Psychological Studies, 8, 11-19.

Domenici, G. (2013b). Successo formativo e inclusione sociale: strategie europee per l'innovazione. Journal of Educational, Cultural and Psychological Studies, 7, 9-14. 
Domenici, G., \& Lucisano, P. (2011). Valutazione, conoscenza, processi decisionali. Dibattito. Journal of Educational, Cultural and Psychological Studies, 3, 147167.

Domenici, G., \& Moretti, G. (a cura di). (2011). Leadership educativa e autonomia scolastica. Il governo dei processi formativi e gestionali nella scuola di oggi. Roma: Armando.

Domingo, P. (2006). Le prove PISA ed INVALSI: possibili conseguenze sulla pratica didattica. Notiziario della Unione Matematica Italiana, 33(11/b), 63-69.

Earley, P., \& Bubb, S. (2004). Leading and managing CPD: Developing people, developing schools. London: Sage - PCP.

Favalli, A. (2011). L'editoria alle prese con i test standardizzati. In V. Spinazzola (a cura di), Tirature 2011. Autori, editori, pubblico (pp. 137-143). Milano: Il Saggiatore - Fondazione Arnoldo - Alberto Mondadori.

Ferraris, M. (2012). Manifesto del nuovo realismo. Roma - Bari: Laterza.

Fondazione Giovanni Agnelli (2010). Rapporto sulla scuola in Italia 2010. Roma Bari: Laterza.

Fondazione Giovanni Agnelli (2011). Rapporto sulla scuola in Italia 2011. Roma Bari: Laterza.

Galliani, L. (2013). Valutare per migliorare il sistema educativo. Il caso INVALSI tra cultura della valutazione e responsabilità politica. Giornale Italiano della Ricerca Educativa, 6(11), 9-15.

Giovannini, M. L. (2007). Considerazioni conclusive. In Ead. (a cura di), Limpatto delle rilevazioni nelle scuole. I punti di vista della pubblica amministrazione e delle istituzioni scolastiche bolognesi (pp. 153-156). Roma: Carocci.

Giovannini, M. L., \& Boni, M. (2010). Verso la valutazione a sostegno dell'apprendimento. Uno studio esplorativo nella scuola primaria. Journal of Educational, Cultural and Psychological Studies, 1, 161-178.

Giovannini, M. L., \& Marcuccio, M. (2007). Un'indagine tra i dirigenti scolastici. In M. L. Giovannini (a cura di), Limpatto delle rilevazioni nelle scuole. I punti di vista della pubblica amministrazione e delle istituzioni scolastiche bolognesi (pp. 57-94). Roma: Carocci.

Grossi, G. (2009). Opinione pubblica e comunicazione politica. Il legame sociale rivisitato. Comunicazione Politica, 1, 45-59.

Hargreaves, A. (2007). Sustainable leadership and development in education: Creating the future, conserving the past. European Journal of Education, 42(2), 223-233.

ISFOL (2013). Le competenze per vivere e lavorare oggi. Principali evidenze dellindagine PIAAC. http://www.trevisosystem-online.com/tvsys/pdf/news/Visco_ Genova_24_settembre2010.pdf.

Karsten, S., \& Visscher, A. (2003). Implicazioni ed effetti della pubblicazione dei risultati scolastici: i casi della Gran Bretagna e della Francia. In N. Bottani \& 
A. Cenerini (a cura di), Una pagella per la scuola. La valutazione tra autonomia e qualità (pp. 229-240). Trento: Erickson.

Leithwood, K., \& Day, C. (2008). The impact of school leadership on pupil outcomes. School Leadership \& Management: Formely School Organisation, 28(1), $1-4$.

Lucisano, P. (2010). Contro la cultura del «giudizio senza critica». Giornale Italiano della Ricerca Educativa, 3(1), 7-8.

Lucisano, P. (2011). Valutare per quale società. Giornale Italiano della Ricerca Educativa, 4(6), 7-9.

Lucisano, P. (2012). Responsabilità sociale, valutazione e ricerca educativa. Giornale Italiano della Ricerca Educativa, Numero speciale, 5, 13-26.

Marcuccio, M. (2010). L'introduzione della Prova Nazionale nell'Esame di Stato al termine del I ciclo d'istruzione: i primi effetti sugli insegnanti di italiano. Journal of Educational, Cultural and Psychological Studies, 5, 27-56.

Mazzoleni, G. (2003). The media and the growth of neo-populism in contemporary democracies. In G. Mazzoleni, J. Stewart, \& B. Horsfield (Eds.), The media and neo-populism (pp. 1-20). London: Praeger.

Moretti, G. (2012). Gli strumenti organizzativi dell'autonomia: il punto di vista degli insegnanti sui documenti. Giornale Italiano della Ricerca Educativa, Numero speciale, 5, 112-129.

OECD (1999). Measuring student knowledge and skills. A new framework for assessment. Paris: OECD.

OECD (2010a). PISA 2009 Results: Learning trends. Paris: OECD.

OECD (2010b). PISA 2009 Results: What makes a school successful? Resources, Policies and Practices, Vol. 4. http://www.pisa.oecd.org/dataoecd/11/16/48852721. pdf.

Pavan De Gregorio, G. (2003). L'indagine IEA PIRLS e la competenza di lettura degli alunni italiani di nove anni. In N. Bottani \& A. Cenerini (a cura di), Una pagella per la scuola. La valutazione tra autonomia e qualità (pp. 339369). Trento: Erickson.

Sammons, P., Gu, Q., Day, C., \& Ko, J. (2011). Exploring the impact of school leadership on pupil outcomes. Results from a study of academically improved and effective schools in England. International Journal of Educational Management, 25(1), 83-101.

Scheerens, J. (2011). Valutazione scolastica interna ed esterna. In J. Scheerens, S. Mosca, \& R. Bolletta (a cura di), Valutare per gestire la scuola. Governance, leadership e qualità educativa (pp. 59-81). Milano: Bruno Mondadori.

Serpieri, R. (2008). Senza leadership, un discorso democratico per la scuola: discorsi e contesti della leadership educativa. Milano: Franco Angeli.

Sestito, P. (2012). La valutazione del valore aggiunto a scuola. Giornale Italiano della Ricerca Educativa, Numero speciale, 5, 27-36. 
Siniscalco, M. T. (2003). La valutazione della competenza di lettura dei quindicenni italiani nell'indagine internazionale OCSE-PISA. In N. Bottani \& A. Cenerini (a cura di), Una pagella per la scuola. La valutazione tra autonomia e qualità (pp. 289-337). Trento: Erickson.

Sylos Labini, F. (2013). Una nota su valutazione e conformismo. Aut Aut, 360, 124-132.

Visscher, A., Bert Dijkstra, A., Karsten, S., \& Veenstra, R. (2003). Una carta dei principi per la pubblicazione delle valutazioni delle scuole. In N. Bottani \& A. Cenerini (a cura di), Una pagella per la scuola. La valutazione tra autonomia e qualità (pp. 241-261). Trento: Erickson.

\section{Riassunto}

L'articolo affronta il tema dell'uso degli esiti delle rilevazioni nazionali e internazionali all'interno dei processi decisionali della "leadership" in ambito scolastico. Il contributo illustra dapprima la rilevanza che assume, anche in Italia, la "leadership" nelle politiche educative evidenziando l'importanza che l'uso degli esiti delle rilevazioni comparative e delle ricerche nazionali e internazionali potrebbe avere per l'esercizio di una "leadership»" orientata a promuovere processi di miglioramento. Il contributo prende poi in esame alcune criticità del contesto italiano che sembrano limitare l'uso efficace degli esiti delle rilevazioni per l'esercizio della "leadership" in campo scolastico: la deresponsabilizzazione dei decisori politici; l'illusoria rassicurazione di scuole, insegnanti e dirigenti; il disimpegno delle scuole al miglioramento della organizzazione didattica e alla qualificazione dei processi di apprendimento in risposta alla sempre piu ampia diffusione di valutazioni esterne percepite come imposte dall'alto. Sono successivamente individuati gli ambiti nei quali l'uso degli esiti delle rilevazioni potrebbe diventare strategico per migliorare la didattica e qualificare i processi di apprendimento. Infine, a questo proposito, viene evidenziata l'importante funzione propositiva che può assumere la "leadership" in campo scolastico nella promozione della cultura del dato e della valutazione.

Parole chiave: Leadership, Miglioramento, Politiche educative, Valutazione, Valutazioni comparative internazionali. 